\title{
Comparison of Sampling Methods in Assessing the Microbiomefrom Patients with Ulcerative Colitis
}

\section{Dan Kim}

Inje University College of Medicine, Busan Paik Hospital

Jun-Young Jung

Inje University College of Medicine, Busan Paik Hospital

Hyun-Seok Oh

Seoul National University

\section{Sam-Ryong Jee}

Inje University College of Medicine, Busan Paik Hospital

\section{Sung Jae Park}

Inje University College of Medicine, Busan Paik Hospital

\section{Sang-Heon Lee}

Inje University College of Medicine, Busan Paik Hospital

Jun-Sik Yoon

Inje University College of Medicine, Busan Paik Hospital

\section{Seung Jung Yu}

Inje University College of Medicine, Busan Paik Hospital

In-Cheol Yoon

Hanyang University College of Medicine

Hong Sub Lee ( $\boldsymbol{\sigma}$ hslee@paik.ac.kr)

Inje University College of Medicine, Busan Paik Hospital

\section{Research Article}

Keywords: Colon lavage, Colonoscopy, Inflammatory bowel disease, Microbiome, Ulcerative Colitis

Posted Date: June 2nd, 2021

DOl: https://doi.org/10.21203/rs.3.rs-540741/v1

License: (-) (1) This work is licensed under a Creative Commons Attribution 4.0 International License. Read Full License 


\section{Abstract}

Background: Dysbiosis of ulcerative colitis (UC) has been frequently investigated using readily accessible stool samples. However, stool samples might insufficiently represent the mucosa-associated microbiome status. We hypothesized that luminal contents including loosely adherent luminal bacteria after bowel preparation may be suitable for diagnosing the dysbiosis of UC.

Methods: This study included 16 patients with UC (9 men and 7 women, mean age: $52.13 \pm 14.09$ years) and 15 sex-and age-matched healthy individuals (8 men and 7 women, mean age: $50.93 \pm 14.11$ years). They donated stool samples before colonoscopy and underwent luminal content aspiration and endoscopic biopsy during the colonoscopy. Then, the composition of each microbiome sample was analyzed by $16 \mathrm{~S}$ rRNA-based next-generation sequencing.

Results: The microbiome between stool, luminal contents, and biopsy was significantly different in alpha and beta diversities. However, a correlation existed between stool and luminal contents in the Procrustes test $(p=0.001)$ and Mantel test $(p=0.0001)$. The stool microbiome was different between patients with UC and the healthy controls. Conversely, no difference was found in the microbiome of luminal content and biopsy samples between the two subject groups. The microbiome of stool and lavage predicted UC, with AUC values of 0.85 and 0.81 , respectively.

Conclusion: The microbiome of stool, luminal contents, and biopsy was significantly different. However, the microbiome of luminal contents during colonoscopy can predict UC, with AUC values of 0.81 . Colonoscopic luminal content aspiration analysis could determine microbiome differences between patients with UC and the healthy control, thereby beneficial in screening dysbiosis via endoscopy.

Trial registration: This trial was registered at http://cris.nih.go.kr.

Registration No.: KCT0003352), Date : 2018-11-13

\section{Background}

Inflammatory bowel diseases (IBD), which include ulcerative colitis (UC) and Crohn's disease (CD), are chronic intestinal diseases.[1] The patients with IBD have a dysbiosis in the intestinal microbiome.[2] The precise mechanism of the disease has not been elucidated, but microbial dysbiosis is one of its major causes.[3] Currently, stool is the most common evaluation sampling measure for assessing gut microbial communities. Although the stool sampling method is readily accessible, people tend to be reluctant to handle their stool, and the sample can be easily contaminated at home.

The gut microbiome consists of two separate populations, namely, the luminal microbiome and mucosa-associated microbiome. A recent study noted marked heterogeneity of the colonic mucosa, indicating that the microbiome between the stool and mucosal biopsy is different.[4] The mucosa-associated microbiota may have more effect on epithelial and mucosal function than luminal bacteria. Hence, fecalysis may inappropriately represent mucosa-associated bacterial populations.[5] However, the optimal sampling of mucosa-associated microbiome has been seldom investigated.

Mucosal tissue collection through endoscopy is the most ideal method for mucosa-associated microbiome,[6] but it is invasive and requires several steps. Therefore, an easy method for evaluating intestinal microorganism imbalance is needed. Recently, colonoscopy has been increasingly used for colon cancer screening. Especially, in patients with UC, colonoscopy was frequently performed for assessing disease activity or treatment response. Aspiration of intestinal fluid through colonoscopy is easy and non-invasive. In general, colonoscopic laxatives exert some influence on the intestinal microbial community.[7] In one study, a colonoscopic laxative significantly reduced the intestinal bacterial count, but in most cases, the count resumed within 14 days.[8] Most of the studies were conducted by collecting stools immediately after colonoscopy rather than examining the fluid aspirated from the colon. According to several studies that applied next-generation sequencing, luminal fluid obtained through colonoscopy is similar to mucosa-associated microbiome.[9] Colonic luminal samples after bowel preparation provide a relatively accurate representation of biopsy microbiome composition and should be considered when biopsy size becomes an issue.[10] Another study using local lavage technique showed similar networks of co-occurring and co-exclusive microbiota, confirming shared features detected in the stool and mucosal lavage compartments.[11, 12] However, analysis of microbiota using local lavage technique in patients with UC has not been reported. 
We hypothesized that luminal contents including loosely adherent luminal bacteria after bowel preparation may be suitable for diagnosing the dysbiosis of UC. Therefore, this study assessed the microbiome differences of colonic luminal samples, biopsy, and stool for all participants. For diagnosing the dysbiosis of UC, all of sampling methods were compared between patients with UC and healthy individuals.

\section{Methods}

This research is a prospective, matched, controlled study that was participated by patients scheduled to receive colonoscopy at teaching hospital in South Korea. The institutional review board of hospital approved the study protocols, following the ethical guidelines of the Declaration of Helsinki. This trial was registered at http://cris.nih.go.kr.

This study examined patients with UC and sex- and age-matched healthy individuals. These participants donated stool samples 3 days before colonoscopy and underwent lavage sampling and endoscopic biopsy during the colonoscopy. We then determined whether the three sampling methods had any difference. All colonoscopies were performed by one board-certified, experienced endoscopist (H.S.L.), who has performed $>5000$ colonoscopies.

The same bowel cleansing product, that is, $2 \mathrm{~L}$ of polyethylene glycol (PEG) plus ascorbate solution (Coolprep ${ }^{\circ}$; Taejoon Pharmaceuticals, Seoul, South Korea), was given to all participants. All of them were scheduled for an evening session (after 14:00). Three days before colonoscopy, they were instructed to refrain from eating foods with high fiber, seeds, sea algae, and mixed grains. One day before the examination, they were asked to eat light dinner and then fast, except drinking water. On the day of the procedure, they were requested to drink the bowel cleansing product $4-8 \mathrm{~h}$ before the examination.

\section{Subjects}

Patients with UC who visited the gastroenterology department of teaching hospital were screened for this study. They were all diagnosed with UC according to the consensus guidelines of the European Crohn's and Colitis Organization.[13] Clinical data such as medical records, blood tests, colonoscopy with biopsy, and radiologic studies were comprehensively reviewed. Furthermore, the clinical activities were evaluated using the Mayo score[14] for UC. Meanwhile, we excluded patients (1) with severe comorbidities, such as cardiovascular diseases, chronic renal diseases, or chronic liver diseases; (2) with mental illnesses; (3)with pregnancy; and (4) with a recent history of gastrointestinal surgery ( $<3$ months).

The healthy-control group consisted of subjects without gastrointestinal diseases or recurrent abdominal symptoms. They were ageand sex-matched with the patient group. To exclude the presence of any recurrent abdominal symptoms and organic diseases, we let them complete the symptom questionnaire, blood tests, and colonoscopy. In addition, all participants were taught on bowel preparation by well-trained nurses from the gastroenterology endoscopy center. Of note, all of them gave written informed consents prior to participation.

\section{Experiments}

\section{Sample collection}

Using a stool sample collector kit (Chun Lab, Inc., Seoul, South Korea), the subjects provided stool samples 3 days before colonoscopy. At the same time, two pellets (approximately $400 \mathrm{mg}$ each) of stool samples were collected from each subject. Each pellet was placed in two sterile plain tubes without any chemical additives. The stool samples were then stored at $-80^{\circ} \mathrm{C}$ before use.

All endoscopic procedures were performed using a white-light endoscope with colonoscopy (CF-H290; Olympus, Tokyo, Japan). During the colonoscopy, approximately $5 \mathrm{~mL}$ of lavage fluid was collected in the sigmoid colon by using a Clear-Hemostat catheter (FMEH0001, Finemedix Co., Daegu, South Korea)(Fig 1). At the same time, single mucosal biopsy was performed at the sigmoid colon by using endoscopic forceps (FB-24K-1; Olympus, Tokyo, Japan). The biopsy sample was kept on ice immediately after collection and then transferred to Chun Lab, Inc. (Seoul, South Korea) on the same day for processing.

\section{Bacterial Community Analysis}




\section{Genomic DNA extraction and amplicon sequencing}

$400 \mathrm{mg}$ of stool, lavage, and biopsy samples were added to $15 \mathrm{~mL}$ of DNA extraction lysis buffer (SDS 4\%, Tris-HCL $50 \mathrm{mM}$, EDTA 50 $\mathrm{mM}, \mathrm{NaCl} 5000 \mathrm{mM}$ ) followed by vigorous homogenization by vortexing for 1 minute. $1.4 \mathrm{~mL}$ of homogenized fecal suspensions were transferred to a $2 \mathrm{~mL}$ eppendorf tube and followed by bead beating for 50 seconds and centrifuging at $14,000 \mathrm{~g}$ for 10 minutes. 200 $\mathrm{uL}$ of supernatants were transferred to 96 well plates were kept at $-25 \otimes C$ until performing PCR. DNA amplification targets V3-V4 regions of the bacterial $16 \mathrm{~S}$ rRNA gene using $341 \mathrm{~F}$ and $805 \mathrm{R}$ primers. PCR products were sequenced using an Illumina Miseq sequencing system (IIlumina, USA) at Chunlab Inc (Seoul, South Korea).

\section{Sequencing data processing and statistical analysis}

EzBioCloud's Microbiome Taxonomic Profiling (MTP) cloud was used to classify sequencing reads to bacterial taxonomy and calculate community composition as previously described.[15] For all subsequent diversity and statistical analyses, the classified reads were randomly rarefied to 8,690 reads, which was the minimum read count among all samples. Alpha-diversity (number of OTUs, Shannon diversity, and Simpson index) indices were calculated in OTU-level and beta-diversity (Bray-Curtis dissimilarity) were generated in genus-level using EzBioCloud's MTP. To see the association between microbial composition variation and host metadata, disease state, and sampling methods, permutational multivariate analysis of variance (PERMANOVA) was conducted using adonis function in vegan R package.[16] PERMANOVA is a geometric partitioning of variation across a multivariate data cloud, defined explicitly in the space of a chosen dissimilarity measure, in response to one or more factors in an analysis of variance design.[17] Pairwise comparisons of community structures between different sampling methods were performed with Procrustes analysis using procrustes and protest function in vegan. The LDA Effect Size (LEfSe; Linear Discriminant Analysis Effect Size) algorithm [18] was used to compare the abundance of taxa among the different groups. Kruskal-Wallis test Pairwise comparisons of community structures between different sampling and Wilcoxon rank-sum test with a significance level of alpha $=0.05$ was used.[19] To evaluate the diagnostic ability of bacterial composition for predicting ulcerative colitis, we constructed L1 penalized least absolute shrinkage and selection operator (LASSO) logistic regression model [20] implemented in 'scikit-learn' package in python.[21] The read counts were centered log-ratio (clr) transformed [22] and the models were built for each sampling methods and their combinations.

\section{Results}

\section{Participant's characteristics}

From September 2018 to January 2019, we prospectively enrolled 16 patients with UC (9 men and 7 women, mean age: $52.13 \pm 14.09$ years) and 15 sex- and age- matched healthy individuals ( 8 men and 7 women, mean age: $50.93 \pm 14.11$ years). However, one healthy man withdrew the consent. Demographic features including age, comorbidities, and body mass index (BMI) were comparable in both subject groups. Current smoking was significantly lower in patients with UC than that in healthy controls ( $0 \%$ vs. $26.7 \% ; p=0.043)$. Most of the patients with UC wherein remission, except for four patients with mild to moderate disease activity (Table 1). 
Table 1

Demographic features of the study subjects



\section{Comparison between sampling methods for all participants}

Out of 93 biopsy samples, five failed to be analyzed because of having a low read count ( $<10,000$ reads). Except the five samples, the averaged taxonomic compositions of stool, lavage, and biopsy were analyzed (Fig. 2A). In the lavage and biopsy samples, the number of microorganisms that was in close contact with the intestines, including Proteobacteria and Akkermansia (a genus in the phylum Verrucomicrobia), increased. Figure 2B illustrates the alpha diversity between the sampling methods. The number of operational taxonomic units in biopsy sampling was significantly higher than that in stool sampling $(p=0.031)$ and lavage sampling $(p<0.006)$. On the basis of the diversity of Shannon and Simpson, the stool sample was significantly different from the biopsy and lavage samples. Figure $2 \mathrm{C}$ shows the beta diversity between sampling methods. All sampling methods in the beta diversity were significantly different from each other.

Table 2 presents the permutational multivariate analysis of variance (PERMANOVA) results between the sampling methods. All sampling methods in PERMANOVA were significantly different from each other. A significant association was also found in the Procrustes test $(0.4901, p=0.001)$ and Mantel test $(p=0.0001)$, indicating a correlation between the stool and wash solution (Fig. 3A). However, the biopsy sample revealed no significant correlation between stool samples (correlation value $=0.2729, p=0.625$ ) and lavage samples (correlation value $=0.2729, p=0.1417$ ) in the Procrustes test $(F i g$. $3 A$ ). The components of the lavage samples 
showed more proportions of microbiome small intestine (Proteobacteria and Enterobacteriaceae), oral and the upper GI tract (Granulicatella, Leptotrichia, Porphyromonasgingivalis, Fusobacterium nucleatum group, Parvimonasmicra, etc.), and respiratory tract (Corynebacterium durum) microbiome..compared to those of the stool samples (Fig. 3B).

Table 2

PERMANOVA of stool, lavage, and biopsy

\begin{tabular}{|lccccc|}
\hline & & df & pseudo-F & $r^{2}$ & $P$-value \\
\hline PERMANOVA & & 2 & 3.589944 & 0.094249 & 0.001 \\
\hline Colon tissue & Lavage & 1 & 2.444345 & 0.056264 & 0.013 \\
\hline & Stool & 1 & 2.435198 & 0.056065 & 0.001 \\
\hline Lavage & Stool & 1 & 5.339986 & 0.087056 & 0.001 \\
\hline df: degrees of freedom; PERMANOVA: permutational multivariate analysis of variance \\
\hline
\end{tabular}

\section{Comparison between patients with UC and the healthy control}

At the phylum level, the relative abundance of Verrucomicrobia was higher in the stool, mucosal, and lavage samples of patients with UC than that of the healthy-control (Fig. 4A,B,C). A significant difference in diversity (number of phylotypes) between the two subject groups was found in the stool sample (Fig. 4D). Conversely, no significant difference was observed in the examination of microorganism diversity in the wash solution and biopsy sample between the two groups. However, the taxonomic distribution of lavage suggests that the differences in diversity may be seen as the number of sample increases.

PERMANOVA confirmed the association between specific factors and the overall microbiome community variation. In the case of stool, UC existence and higher disease activity were distinguished. Lavage was significantly related to current smoking, intestinal surgery, and intestinal symptoms. Furthermore, colon tissue was significantly related to BMI, surgery, order number, and sampling date (Table 3). 
Table 3

Metadata association with microbiota composition variation (Bray-Curtis distance, Genus level) by permutational multivariate analysis of variance

\begin{tabular}{|c|c|c|c|c|c|c|c|c|c|c|}
\hline & & Stool & & & Lavage & & & Colontissu & & \\
\hline & df & pseudo-F & $r^{2}$ & $P$-value & pseudo-F & $r^{2}$ & $P$-value & pseudo-F & $r^{2}$ & $P$-value \\
\hline Birthmonth & 1 & 0.496807 & 0.017434 & 0.963 & 0.30401 & 0.010741 & 0.988 & 0.893998 & 0.064344 & 0.587 \\
\hline Bristol stool & 1 & 1.615966 & 0.054564 & 0.09 & 1.126012 & 0.03866 & 0.338 & 0.591071 & 0.04349 & 0.923 \\
\hline Complication & 1 & 0.978535 & 0.070003 & 0.404 & 1.660764 & 0.11328 & 0.132 & 0.835214 & 0.094532 & 0.66 \\
\hline Drinking & 1 & 0.904672 & 0.031298 & 0.532 & 0.629747 & 0.021996 & 0.741 & 0.810813 & 0.058709 & 0.709 \\
\hline Extraintestinal & 1 & 1.317045 & 0.091991 & 0.133 & 2.485206 & 0.160489 & 0.02 & 0.928086 & 0.103951 & 0.494 \\
\hline Fermentedfood & 1 & 0.77691 & 0.026998 & 0.666 & 1.408026 & 0.047879 & 0.159 & 1.137492 & 0.080459 & 0.284 \\
\hline Surgery history & 1 & 0.850101 & 0.029466 & 0.567 & 1.764313 & 0.059276 & 0.079 & 1.232748 & 0.086613 & 0.191 \\
\hline Age & 1 & 1.33408 & 0.045479 & 0.169 & 1.187432 & 0.040683 & 0.266 & 0.671462 & 0.049114 & 0.876 \\
\hline Birthyear & 1 & 1.341552 & 0.045722 & 0.17 & 1.239168 & 0.04238 & 0.226 & 0.662354 & 0.04848 & 0.899 \\
\hline BMI & 1 & 1.382341 & 0.047047 & 0.17 & 0.595062 & 0.02081 & 0.798 & 1.964587 & 0.131282 & 0.015 \\
\hline Hostcategory & 1 & 2.348238 & 0.077376 & 0.013 & 1.072956 & 0.036906 & 0.31 & 1.07396 & 0.076308 & 0.336 \\
\hline Height & 1 & 1.610117 & 0.054377 & 0.085 & 0.387902 & 0.013664 & 0.954 & 0.483476 & 0.035857 & 0.981 \\
\hline Sex & 1 & 0.943593 & 0.032601 & 0.467 & 0.733253 & 0.025519 & 0.651 & 0.707142 & 0.051589 & 0.852 \\
\hline Weight & 1 & 1.24015 & 0.042413 & 0.237 & 0.591836 & 0.020699 & 0.796 & 1.821861 & 0.122917 & 0.025 \\
\hline Hypertension & 1 & 0.428682 & 0.015079 & 0.986 & 0.724504 & 0.025222 & 0.662 & 0.715157 & 0.052144 & 0.839 \\
\hline LesionE1, E23 & 1 & 0.833943 & 0.060282 & 0.646 & 0.556671 & 0.041062 & 0.865 & 0.772521 & 0.088061 & 0.731 \\
\hline $\begin{array}{l}\text { LesionE1, E2, } \\
\text { E3 }\end{array}$ & 2 & 1.534195 & 0.203631 & 0.053 & 0.702836 & 0.104857 & 0.775 & 1.097036 & 0.23864 & 0.359 \\
\hline Medication & 1 & 0.662934 & 0.023129 & 0.789 & 0.6435 & 0.022466 & 0.727 & 0.729636 & 0.053143 & 0.818 \\
\hline Ordernumber & 8 & 1.273526 & 0.326669 & 0.099 & 1.151589 & 0.304928 & 0.215 & 1.450733 & 0.446279 & 0.02 \\
\hline Pastdisease & 9 & 0.751518 & 0.252718 & 0.882 & 0.887925 & 0.285493 & 0.683 & 0.818017 & 0.44995 & 0.899 \\
\hline Period & 1 & 0.954267 & 0.068385 & 0.507 & 1.564211 & 0.107401 & 0.122 & 0.927049 & 0.103847 & 0.529 \\
\hline Probiotics & 1 & 0.632417 & 0.022087 & 0.839 & 0.587319 & 0.020545 & 0.815 & 1.1144 & 0.078955 & 0.289 \\
\hline Probiotics & 1 & 0.763851 & 0.026556 & 0.692 & 0.634697 & 0.022165 & 0.729 & 1.329072 & 0.092754 & 0.139 \\
\hline Samplingdate & 8 & 1.273526 & 0.326669 & 0.11 & 1.151589 & 0.304928 & 0.228 & 1.450733 & 0.446279 & 0.032 \\
\hline Smoking & 1 & 0.895662 & 0.030996 & 0.489 & 2.138451 & 0.070954 & 0.041 & 0.859489 & 0.062014 & 0.663 \\
\hline Stoolcolor & 3 & 1.27103 & 0.1279 & 0.178 & 0.880472 & 0.092224 & 0.592 & 1.086841 & 0.22864 & 0.331 \\
\hline Stooltype & 5 & 1.000135 & 0.172433 & 0.46 & 0.722303 & 0.130797 & 0.91 & 1.107801 & 0.380976 & 0.275 \\
\hline Sugarydrink & 1 & 1.472612 & 0.049965 & 0.117 & 0.863336 & 0.029911 & 0.506 & 1.126826 & 0.079765 & 0.286 \\
\hline Sugarysnack & 1 & 0.816938 & 0.028349 & 0.628 & 1.283086 & 0.043817 & 0.248 & 0.799636 & 0.057946 & 0.727 \\
\hline Surgery & 5 & 1.509554 & 0.239249 & 0.053 & 1.723835 & 0.264236 & 0.004 & 1.419619 & 0.279107 & 0.046 \\
\hline Active UC & 1 & 2.21661 & 0.073357 & 0.024 & 1.015076 & 0.034984 & 0.346 & 1.061837 & 0.075512 & 0.358 \\
\hline
\end{tabular}




\begin{tabular}{|c|c|c|c|c|c|c|c|c|c|c|}
\hline & & Stool & & & Lavage & & & Colontissu & & \\
\hline UC activity & 1 & 1.965438 & 0.131332 & 0.046 & 0.693475 & 0.050643 & 0.692 & 0.710533 & 0.081572 & 0.811 \\
\hline
\end{tabular}

Modeling confirmed that microorganisms in each microbiome can distinguish diseases. Stool and lavage predicted UC, obtaining the AUC values of 0.85 and 0.81 , respectively (Fig. $5 A, B)$. The accuracy $(A U C=0.88)$ increases when using the data from stool and lavage samples.(Fig. 5C)

In the case of stool, we found that facultative anaerobes such as Enterococcus and Lactobacillus are UC-related biomarkers, and several genera of Bacteroidetes (Prevotella, Bacteroides, etc.) and Clostridiales are the healthy-control markers (Fig. 6A). In the case of lavage, Staphylococcus and Leuconostocaceae are the disease markers, whereas Prevotella, Alistipes, and Desulfovibrio are the healthy-control markers (Fig. 6B). Therefore, lavage has fewer markers than stool, and microorganisms different from those in the stool are disease and healthy-control markers.

\section{Discussion}

Despite the reduction of beneficial microbes, expansion of pathobionts, and the reduced microbial diversity, which all indicate IBD, the dysbiosis remains inconsistent, and the composition of microbiome varies.[23] The reason for variation may be because of heterogeneity in the sampling method and the disease status of the subject.

In this study, although lavage fluid and stool showed some correlation, all three samples (i.e., lavage, stool, and biopsy samples) were significantly different from each other. The results are consistent with those in previous studies. The microbial communities of multiple biopsy sites within the mouth, stomach, duodenum, and colon were largely distinct from those in stool.[4, 24, 25] In addition, the microbiome variation by colonic biopsy between different colonic regions showed the significant effect by sampling site on the abundance of bacterial taxa.[26] In our study, the biopsy and lavage samples revealed that Firmicutes and Bacteroidetes were less abundant, with the concomitant increase of Proteobacteria and fusobacteria. A study participated by Korean patients with CD showed a similar distribution to our study.[27] Thus, biopsy reflects mucosa-associated microbiome, whereas the stool reflects the luminal microbiota in the distal large intestine.

Luminal contents after bowel preparation provide the closest microbiome of the biopsy sample.[9] Colonic lavage samples also provide a relatively accurate representation of biopsy microbiome composition.[10] Bowel preparation appears sufficient to cleanse the lumen to ensure that the luminal contents sampled at colonoscopy-being derived from mucus-adherent microbes rather than the bulk stool stream-contain a microbial community more similar to that of the biopsy samples. The stool material obtained during colonoscopy could be more representative of the mucosal layer. Hence, a noninvasive lavage analysis would be a good alternative method to biopsy. However, in this study, biopsy and lavage samples had no association. Five biopsy samples were not analyzed in this study because of low read counts. The reason is that biopsy procedure is too invasive to acquire enough amount of tissue for microbiome analysis. Furthermore, bowel cleansing washed the entire intestine; thus, the post-bowel preparation fluid could contain loosely attached mucosa-associated microbiome and the whole part of luminal microbiome from the mouth to the colon. The lavage microbes would originate from the whole intestine, oral cavity, and respiratory tract; therefore, colonic lavage samples may have some variances. Meanwhile, another method using local lavage and brush technique for each intestinal part has been reported but still requires validation. $[11,12,28]$

Most of the studies analyzing the mucosa-associated microbiome performed biopsy sampling after bowel cleansing. In general, colonoscopy preparations can result in multiple negative effects on the intestinal microbiome. Standard bowel preparation can alter the diversity of mucosa-associated microbiota.[29] At the class level, Proteobacteria and Coriobacteria increase, whereas Clostridia significantly decrease after colonoscopy.[30] In normal individuals, a high-volume PEG bowel cleansing preparation has a long-lasting effect on the gut microbiota composition and homeostasis. The short-term effects of bowel preparation may be because most of the bacteria are being washed out in a nondiscriminatory manner, reducing the low-abundance taxonomic groups to levels below detection; on average, bowel preparation with PEG can reduce bacterial load.[7] In fresh stool samples and distal colonic mucosal biopsies collected from 24 healthy subjects before and during a flexible sigmoidoscopy of an unprepared bowel, stool samples obtained a significantly higher diversity than the mucosal samples.[31] More endoscopic studies of an unprepared bowel would be necessary to validate the biopsy method. 
The stool samples showed significant differences in diversity between UC and healthy controls. Conversely, the microorganism diversity examination in the colonic lavage and biopsy samples had no significant difference between these two groups. However, the sample size in this study is small to acquire accurate verification. Therefore, we cannot conclude that lavage does not differ between the two groups, considering that the association between intestinal microorganisms and metadata does not consistently appear as a change in the overall population. The change of one or some combinations of specific microorganisms may be a feature that distinguishes UC, and in this case, it can be confirmed by modeling such as machine learning. In this study, stool and lavage predicted UC, obtaining the AUC values of 0.85 and 0.81 , respectively, for accuracy. Considering that the accuracy of the model is relatively high (AUC > 0.8), lavage also has a diagnostic potential to verify UC. In another study using biopsy and stool, terminal ileum biopsies performed best (AUC $=0.85$ ), closely matched by the rectum biopsies (AUC $=0.78$ ). The classifier based on the stool samples collected during the diagnosis performed less satisfactorily (AUC $=0.66$ ), with low consistency.[6] Thus, microbiome analysis using colonic lavage can distinguish diseases.

Moreover, facultative anaerobes such as Enterococcus and Lactobacillus are UC-related biomarkers in the case of stool. This result correlates with the shift of bacterial communities from obligate to facultative anaerobes, strongly suggesting a disruption in anaerobiosis and pointing to a potential role for oxygen in intestinal dysbiosis.[32] Decreased influence of obligate anaerobes and increased influence of facultative anaerobes and some aerobic bacterial communities generally happen in UC.[33] In addition, Bifidobacterium and the Lactobacillus group were increased in patients with active IBD.[34] Generally, in subjects with infectious diarrhea, Proteobacteria and Enterobacteriaceae increase and lactobacilli decrease.[35] In the case of lavage, Staphylococcus and Leuconostocaceae are the disease markers. Staphylococcus could be one of the candidates involved in IBD pathogenesis.

Staphylococcus aureus can be detected in a UC-affected colon.[36] The species of the Enterobacteriaceae family only increased in patients with CD; examples were Escherichia/Shigella species, which commonly invade the gut mucosal epithelium, causing bloody diarrhea and colonic ulceration.[37] The stool bacteria from patients with UC could cause stronger inflammatory responses than those from healthy controls.[38]

A typical gut microbiota in patients with IBD is characterized by a decrease in stool bacteria such as Firmicutes and Bacteroidetes and an increase in Proteobacteria. However, this finding was not significant in this study. Enrolled patients had inactive UC. Hence, their gut microbiome tended to be similar to that of healthy individuals, suggesting that the stool microbiota has different roles in UC pathophysiology.[39]

Meanwhile, this study has several limitations. First, the lavage procedure was not performed at each local intestinal part. Some lavage fluid in the sigmoid colon may not represent the whole intestine microbiome status. Nonetheless, the colonic lavage sample can show the whole gastrointestinal mucosa-associated microbiome status. Second, our sample size is small; we only enrolled a limited number to extensively analyze each stool, lavage, and biopsy sample. Third, our study did not demonstrate longitudinal microbiome variation between patients with UC and the healthy control. Hence, further studies with more subjects and longitudinal samplings are necessary.

\section{Conclusion}

In conclusion, the sampling methods of analyzing the colon microbiome differ from each other. Colonoscopic lavage analysis can determine microbiome differences between patients with UC and the healthy control, thereby beneficial in screening dysbiosis via endoscopy.

\section{Abbreviations}

UC(ulcerative colitis), AUC(area under receiver operating characteristic curve)

\section{Declarations}

\section{Consent for publication:}

Written informed consent was obtained from patients who participated in this study.

\section{Availability of data and materials:}




\section{Funding:}

This work was supported by the National Research Foundation of Korea(NRF) grant funded by the Korea government(MSIT) (No. 2020R1G1A100923411).

This work was supported by the 2019 Inje University research grant.

\section{Acknowledgements:}

Not applicable.

\section{Authors' information:}

Corresponding author: Hong Sub Lee, MD, PhD

Department of Internal Medicine

Inje University College of Medicine, Busan Paik Hospital, Busan, Korea.

75 Bokji-ro, Busanjin-gu, Busan 47392, Korea

Tel: +82-51-890-6989 Fax: +82-51-892-0273

E-mail:hslee@paik.ac.kr

\section{Author Contributions:}

Concept - H.S.L.,I.C.Y.; Design - H.S.L.,I.C.Y.; Supervision - S.H.L.,J.S.Y.,S.J.P; Resource - H.S.L.; Materials - H.S.L., D.K.; Data Collection and/or Processing - H.S.L.,I.C.Y.,H.S.O.,D.K.; Analysis and/or Interpretation - H.S.L., H.S.O.,J.Y.J.; Literature Search -

H.S.L.,S.J.Y,H.S.O.,J.Y.J; Writing - H.S.L; Critical Reviews - S.J.Y.,S.R.J.,S.H.L.,J.S.Y., S.J.P

\section{Ethics Committee Approval:}

Ethics committee approval was received for this study from Institutional Review Board of the Myongji hospital (Decision No: MJH 2018-08-020).

\section{Peer-review:}

Externally peer-reviewed.

\section{Competing interests:}

The authors declare that they have no competing interests.

\section{References}

1. Matsuoka K, Kanai T. The gut microbiota and inflammatory bowel disease. Seminars in immunopathology. 2015;37(1):47-55. Epub 2014/11/26. doi: 10.1007/s00281-014-0454-4. PubMed PMID: 25420450; PubMed Central PMCID: PMCPMC4281375. 
2. Lavelle A, Lennon G, Winter DC, O'Connell PR. Colonic biogeography in health and ulcerative colitis. Gut microbes. 2016;7(5):43542. Epub 2016/09/24. doi: 10.1080/19490976.2016.1216748. PubMed PMID: 27662587; PubMed Central PMCID: PMCPMC5154370.

3. Sartor RB, Wu GD. Roles for Intestinal Bacteria, Viruses, and Fungi in Pathogenesis of Inflammatory Bowel Diseases and Therapeutic Approaches. Gastroenterology. 2017;152(2):327-39.e4. Epub 2016/10/23. doi: 10.1053/j.gastro.2016.10.012. PubMed PMID: 27769810; PubMed Central PMCID: PMCPMC5511756.

4. Zhang Z, Geng J, Tang X, Fan H, Xu J, Wen X, et al. Spatial heterogeneity and co-occurrence patterns of human mucosalassociated intestinal microbiota. The ISME journal. 2014;8(4):881-93. Epub 2013/10/18. doi: 10.1038/ismej.2013.185. PubMed PMID: 24132077; PubMed Central PMCID: PMCPMC3960530.

5. Sartor RB. Gut microbiota: Optimal sampling of the intestinal microbiota for research. Nature reviews Gastroenterology \& hepatology. 2015;12(5):253-4. Epub 2015/03/25. doi: 10.1038/nrgastro.2015.46. PubMed PMID: 25802025.

6. Gevers D, Kugathasan S, Denson LA, Vazquez-Baeza Y, Van Treuren W, Ren B, et al. The treatment-naive microbiome in new-onset Crohn's disease. Cell host \& microbe. 2014;15(3):382-92. Epub 2014/03/19. doi: 10.1016/j.chom.2014.02.005. PubMed PMID: 24629344; PubMed Central PMCID: PMCPMC4059512.

7. Jalanka J, Salonen A, Salojarvi J, Ritari J, Immonen O, Marciani L, et al. Effects of bowel cleansing on the intestinal microbiota. Gut. 2015;64(10):1562-8. Epub 2014/12/21. doi: 10.1136/gutjnl-2014-307240. PubMed PMID: 25527456.

8. O'Brien CL, Allison GE, Grimpen F, Pavli P. Impact of colonoscopy bowel preparation on intestinal microbiota. PloS one. 2013;8(5):e62815. Epub 2013/05/08. doi: 10.1371/journal.pone.0062815. PubMed PMID: 23650530; PubMed Central PMCID: PMCPMC3641102.

9. Shaw AG, Black N, Rushd A, Sim K, Randell P, Kroll JS, et al. Assessing the Colonic Microbiota in Children: Effects of Sample Site and Bowel Preparation. Journal of pediatric gastroenterology and nutrition. 2017;64(2):230-7. Epub 2016/04/14. doi: 10.1097/mpg.0000000000001233. PubMed PMID: 27070657.

10. Watt E, Gemmell MR, Berry S, Glaire M, Farquharson F, Louis P, et al. Extending colonic mucosal microbiome analysis-assessment of colonic lavage as a proxy for endoscopic colonic biopsies. Microbiome. 2016;4(1):61. Epub 2016/11/26. doi: 10.1186/s40168016-0207-9. PubMed PMID: 27884202; PubMed Central PMCID: PMCPMC5123352.

11. Li X, LeBlanc J, Truong A, Vuthoori R, Chen SS, Lustgarten JL, et al. A metaproteomic approach to study human-microbial ecosystems at the mucosal luminal interface. PloS one. 2011;6(11):e26542. Epub 2011/12/02. doi: 10.1371/journal.pone.0026542. PubMed PMID: 22132074; PubMed Central PMCID: PMCPMC3221670.

12. Tong M, Li X, Wegener Parfrey L, Roth B, Ippoliti A, Wei B, et al. A modular organization of the human intestinal mucosal microbiota and its association with inflammatory bowel disease. PloS one. 2013;8(11):e80702. Epub 2013/11/22. doi: 10.1371/journal.pone.0080702. PubMed PMID: 24260458; PubMed Central PMCID: PMCPMC3834335.

13. Dignass A, Eliakim R, Magro F, Maaser C, Chowers Y, Geboes K, et al. Second European evidence-based consensus on the diagnosis and management of ulcerative colitis part 1: definitions and diagnosis. J Crohns Colitis. 2012;6(10):965-90. Epub 2012/10/09. doi: 10.1016/j.crohns.2012.09.003. PubMed PMID: 23040452.

14. Lewis JD, Chuai S, Nessel L, Lichtenstein GR, Aberra FN, Ellenberg JH. Use of the noninvasive components of the Mayo score to assess clinical response in ulcerative colitis. Inflammatory bowel diseases. 2008;14(12):1660-6. Epub 2008/07/16. doi: 10.1002/ibd.20520. PubMed PMID: 18623174; PubMed Central PMCID: PMCPMC2597552.

15. Yoon S-H, Ha S-M, Kwon S, Lim J, Kim Y, Seo H, et al. Introducing EzBioCloud: a taxonomically united database of 16S rRNA gene sequences and whole-genome assemblies. International journal of systematic and evolutionary microbiology. 2017;67(5):1613.

16. Dixon P. VEGAN, a package of R functions for community ecology. Journal of Vegetation Science. 2003;14(6):927-30.

17. Anderson MJ. Permutational Multivariate Analysis of Variance (PERMANOVA). Wiley StatsRef: Statistics Reference Online2017. p. 1-15.

18. Segata N, Izard J, Waldron L, Gevers D, Miropolsky L, Garrett WS, et al. Metagenomic biomarker discovery and explanation. Genome biology. 2011;12(6):R60.

19. Emmert-Streib F, Dehmer M. Large-Scale Simultaneous Inference with Hypothesis Testing: Multiple Testing Procedures in Practice. Machine Learning and Knowledge Extraction. 2019;1(2):653-83. PubMed PMID: doi:10.3390/make1020039.

20. Tibshirani R. Regression shrinkage and selection via the lasso. Journal of the Royal Statistical Society: Series B (Methodological). 1996;58(1):267-88.

Page $11 / 18$ 
21. Pedregosa F, Varoquaux G, Gramfort A, Michel V, Thirion B, Grisel O, et al. Scikit-learn: Machine learning in Python. the Journal of machine Learning research. 2011;12:2825-30.

22. Aitchison J. The Analysis of Compositional Data (London. Chapman and. 1986.

23. Petersen C, Round JL. Defining dysbiosis and its influence on host immunity and disease. Cellular microbiology. 2014;16(7):102433. Epub 2014/05/07. doi: 10.1111/cmi.12308. PubMed PMID: 24798552; PubMed Central PMCID: PMCPMC4143175.

24. Stearns JC, Lynch MD, Senadheera DB, Tenenbaum HC, Goldberg MB, Cvitkovitch DG, et al. Bacterial biogeography of the human digestive tract. Scientific reports. 2011;1:170. Epub 2012/02/23. doi: 10.1038/srep00170. PubMed PMID: 22355685; PubMed Central PMCID: PMCPMC3240969.

25. Wang M, Ahrne S, Jeppsson B, Molin G. Comparison of bacterial diversity along the human intestinal tract by direct cloning and sequencing of 16 S rRNA genes. FEMS microbiology ecology. 2005;54(2):219-31. Epub 2005/12/08. doi: 10.1016/j.femsec.2005.03.012. PubMed PMID: 16332321.

26. de Cárcer DA, Cuív PÓ, Wang T, Kang S, Worthley D, Whitehall V, et al. Numerical ecology validates a biogeographical distribution and gender-based effect on mucosa-associated bacteria along the human colon. The ISME journal. 2011;5(5):801-9. doi: 10.1038/ismej.2010.177.

27. Eun CS, Kwak M-J, Han DS, Lee AR, Park DI, Yang S-K, et al. Does the intestinal microbial community of Korean Crohn's disease patients differ from that of western patients? BMC Gastroenterology. 2016;16(1):28. doi: 10.1186/s12876-016-0437-0.

28. Nishino K, Nishida A, Inoue R, Kawada Y, Ohno M, Sakai S, et al. Analysis of endoscopic brush samples identified mucosaassociated dysbiosis in inflammatory bowel disease. Journal of gastroenterology. 2018;53(1):95-106. Epub 2017/08/31. doi: 10.1007/s00535-017-1384-4. PubMed PMID: 28852861.

29. Harrell L, Wang Y, Antonopoulos D, Young V, Lichtenstein L, Huang Y, et al. Standard Colonic Lavage Alters the Natural State of Mucosal-Associated Microbiota in the Human Colon. PloS one. 2012;7(2):e32545. doi: 10.1371/journal.pone.0032545.

30. Drago L, Toscano M, De Grandi R, Casini V, Pace F. Persisting changes of intestinal microbiota after bowel lavage and colonoscopy. European journal of gastroenterology \& hepatology. 2016;28(5):532-7. Epub 2016/03/26. doi: 10.1097/meg.0000000000000581. PubMed PMID: 27015015.

31. Ringel Y, Maharshak N, Ringel-Kulka T, Wolber EA, Sartor RB, Carroll IM. High throughput sequencing reveals distinct microbial populations within the mucosal and luminal niches in healthy individuals. Gut microbes. 2015;6(3):173-81. Epub 2015/04/29. doi: 10.1080/19490976.2015.1044711. PubMed PMID: 25915459; PubMed Central PMCID: PMCPMC4615648.

32. Rigottier-Gois L. Dysbiosis in inflammatory bowel diseases: the oxygen hypothesis. The ISME journal. 2013;7(7):1256-61. Epub 2013/05/17. doi: 10.1038/ismej.2013.80. PubMed PMID: 23677008; PubMed Central PMCID: PMCPMC3695303.

33. Walujkar SA, Dhotre DP, Marathe NP, Lawate PS, Bharadwaj RS, Shouche YS. Characterization of bacterial community shift in human Ulcerative Colitis patients revealed by Illumina based 16S rRNA gene amplicon sequencing. Gut pathogens. 2014;6(1):22.

34. Wang W, Chen L, Zhou R, Wang X, Song L, Huang S, et al. Increased proportions of Bifidobacterium and the Lactobacillus group and loss of butyrate-producing bacteria in inflammatory bowel disease. Journal of clinical microbiology. 2014;52(2):398-406. Epub 2014/01/31. doi: 10.1128/jcm.01500-13. PubMed PMID: 24478468; PubMed Central PMCID: PMCPMC3911339.

35. Pop M, Walker AW, Paulson J, Lindsay B, Antonio M, Hossain MA, et al. Diarrhea in young children from low-income countries leads to large-scale alterations in intestinal microbiota composition. Genome biology. 2014;15(6):R76. Epub 2014/07/06. doi: 10.1186/gb-2014-15-6-r76. PubMed PMID: 24995464; PubMed Central PMCID: PMCPMC4072981.

36. M. Chiba SHMKMTTFMISW. Staphylococcus aureus in Inflammatory Bowel Disease. Scandinavian journal of gastroenterology. 2001;36(6):615-20. doi: 10.1080/00365520117407.

37. Anderson M, Sansonetti PJ, Marteyn BS. Shigella Diversity and Changing Landscape: Insights for the Twenty-First Century. Front Cell Infect Microbiol. 2016;6:45-. doi: 10.3389/fcimb.2016.00045. PubMed PMID: 27148494.

38. Wang S, Yao L, Liu Y. Fecal microbiome from patients with ulcerative colitis is potent to induce inflammatory responses. International immunopharmacology. 2018;59:361-8. Epub 2018/04/24. doi: 10.1016/j.intimp.2018.04.014. PubMed PMID: 29684823.

39. Andoh A, Imaeda H, Aomatsu T, Inatomi O, Bamba S, Sasaki M, et al. Comparison of the fecal microbiota profiles between ulcerative colitis and Crohn's disease using terminal restriction fragment length polymorphism analysis. Journal of gastroenterology. 2011;46(4):479-86. Epub 2011/01/22. doi: 10.1007/s00535-010-0368-4. PubMed PMID: 21253779.

Page 12/18 


\section{Figures}

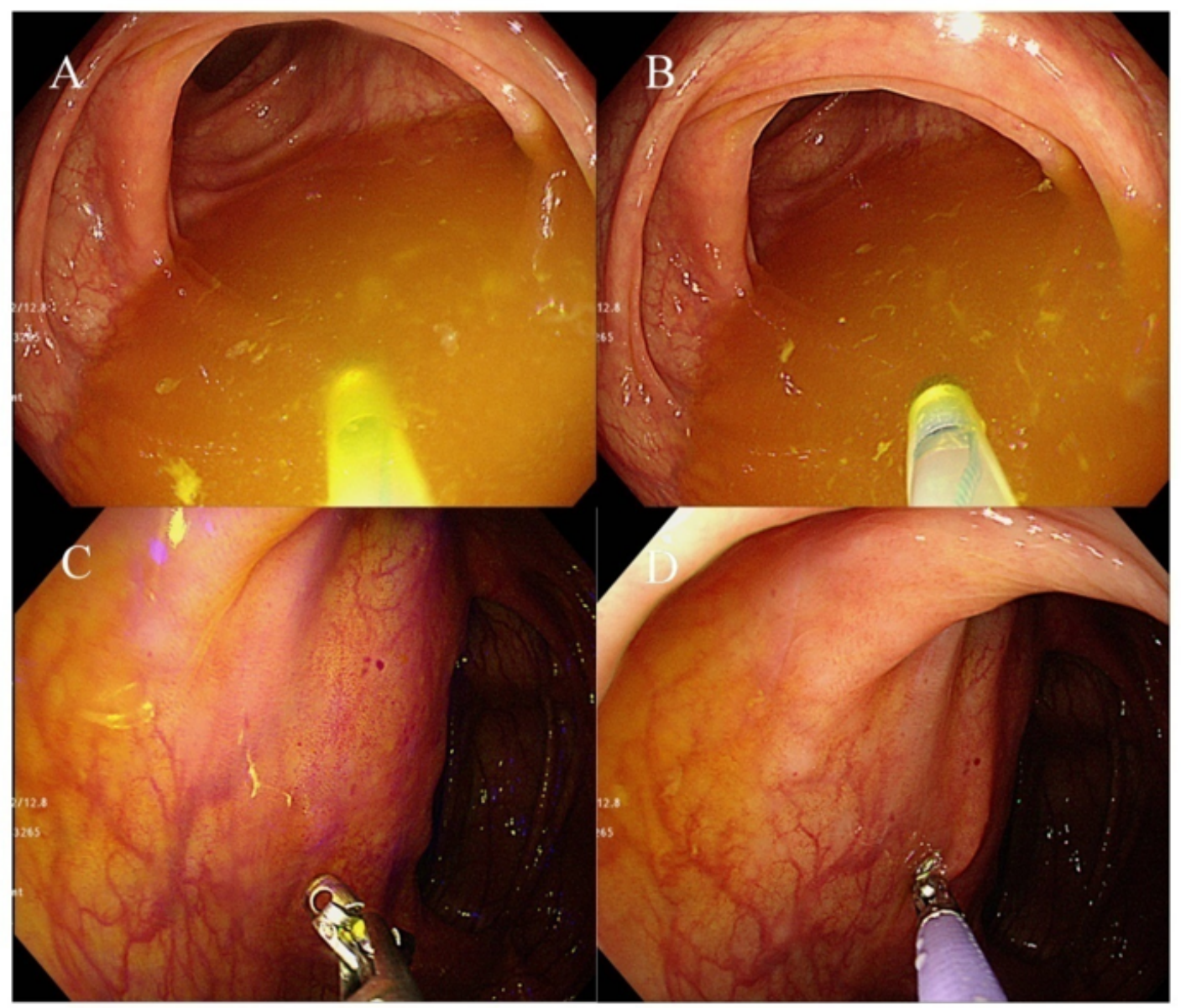

\section{Figure 1}

Method to sample the lavage fluid and biopsy. (A, B) About $5 \mathrm{ml}$ of colonic lavage fluid in sigmoid colon was collected by using a ClearHemostat catheter. (C, D) After colonic lavage sampling, the single mucosal biopsy was done at S colon with endoscopic forceps. 
Fig 2A

Phylum
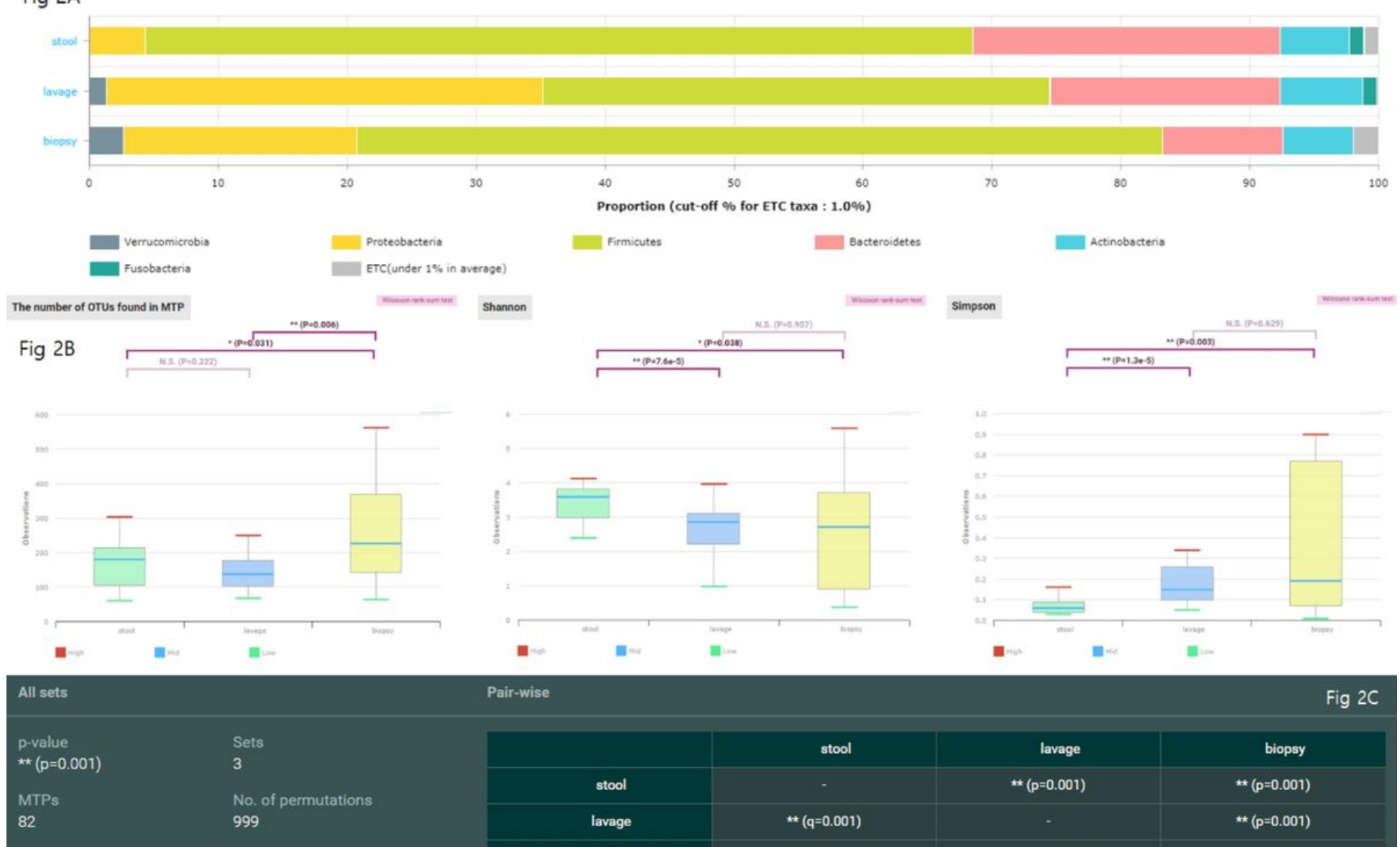

Pair-wise

Fig $2 C$

\section{Figure 2}

Comparison between sampling methods (A) averaged taxonomic compositions at phylum level for stool, lavage, and biopsy sample.

(B) Boxplot of Alpha-diversity.in the three study groups stool, lavage, biopsy by means of observed Operational Taxonomic Units (OTUs), Shannon, and Simpson indexes. Plotted in the graphic are the inter-quartile ranges (IQRs) and boxes, medians (lines in the boxes), and lowest and highest values for the first and third quartiles. Each phenotypic category is identified by colors, as indicated on the right side of the figure. Every sample is represented by a colored dot. Solid lines and asterisks indicate that the numbers of OTUs in biopsy sampling was significantly higher than stool sample $(p=0.031)$ and lavage $(p<0.006)$. In the diversity of Shannon and Simpson, stool sample was significantly different with biopsy and lavage sample. (C) Boxplot of Beta diversity (stool, lavage, biopsy). All sampling method in the Beta-diversity was significantly different with each other. 
A

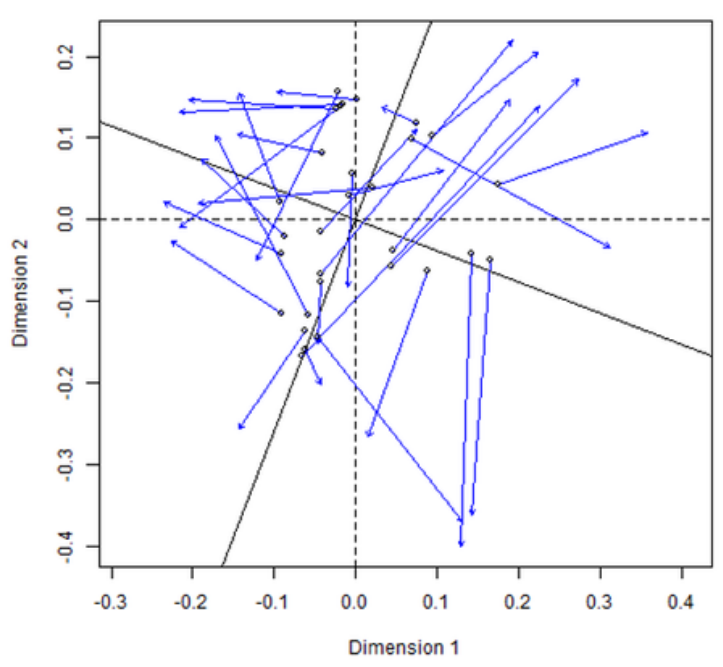

B

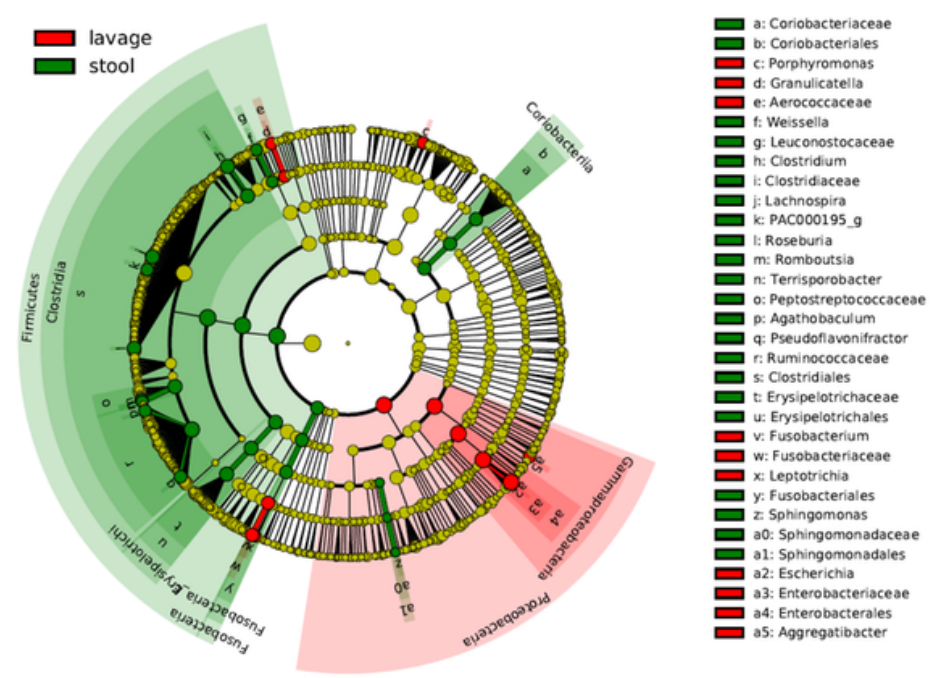

\section{Figure 3}

Comparison between colonic lavage and biopsy samples. (A) Procrustes visualizations of non-metric multidimensional scaling plots compared at the phylum and family levels for stool vs. lavage. (B) Cladogram generated by LEfSe indicating differences in taxa between lavage group and stool group. Each successive circle represents a phylogenetic level (phylum, class, order, family, genus). Regions in red indicate taxa enriched in lavage group while regions in green indicate taxa enriched in stool group. Different taxa (at family and order level) are listed on the right side of the cladogram. 

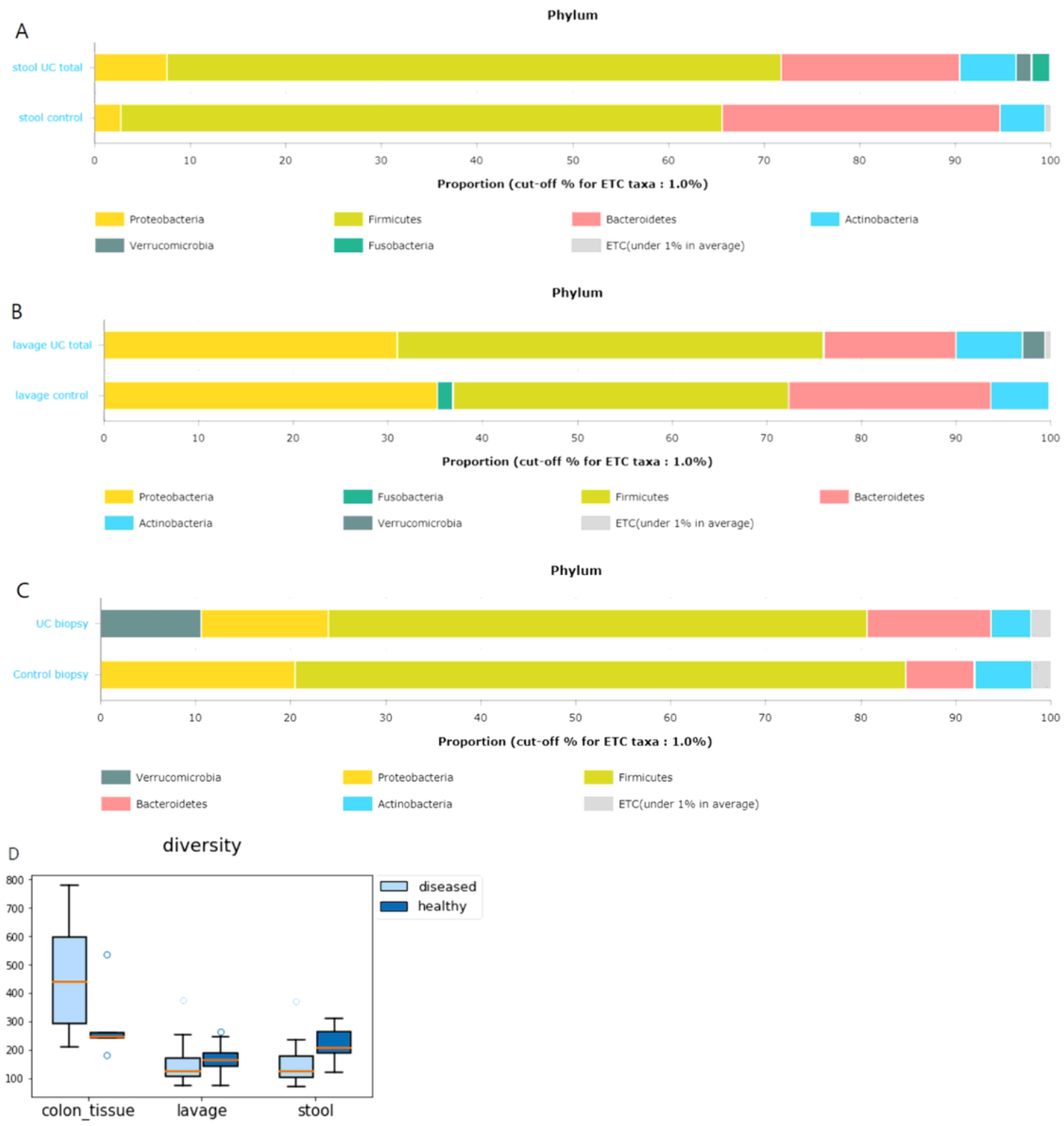

\section{Figure 4}

The comparison between patients with UC and control. (A) Averaged taxonomic compositions at phylum level for stool in patients with UC and control. (B) Averaged taxonomic compositions at phylum level for colonic lavage in patients with UC and control. (C) Averaged taxonomic compositions at phylum level for biopsy in patients with UC and control. (D) Significant differences in diversity (number of phylotypes) between UC and healthy controls was found in stool sample. 

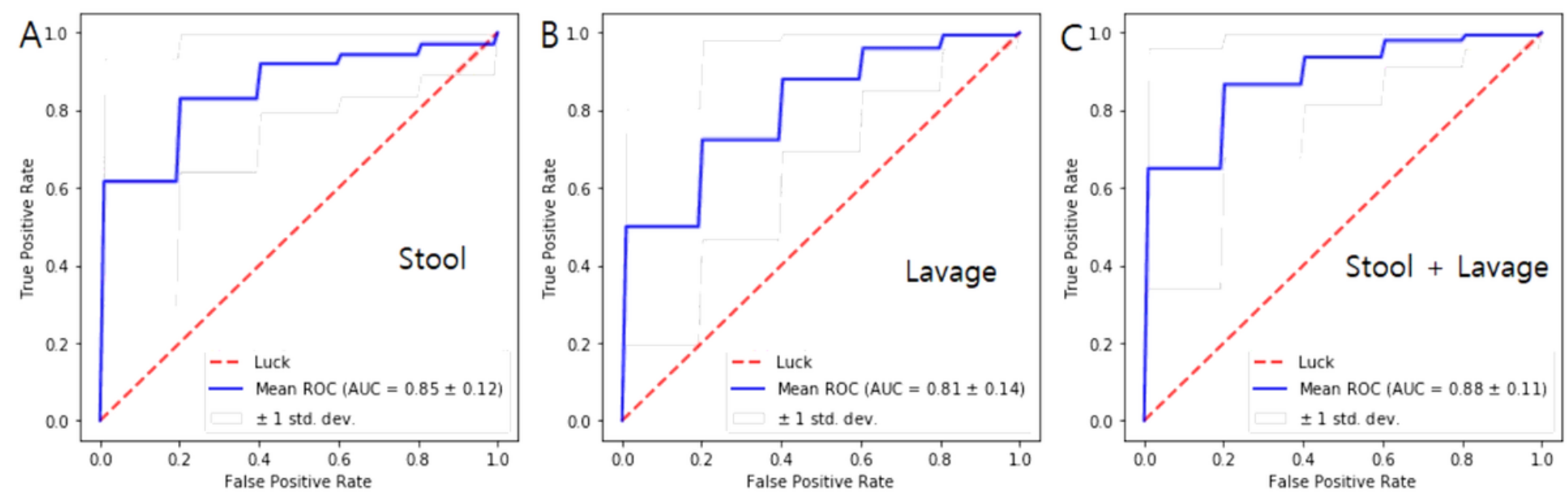

Figure 5

Least absolute shrinkage and selection operator (LASSO) for prediction of ulcerative colitis (UC) means Receiver Operating Characteristic (ROC) curve for UC diagnosis (A) Stool predicted UC in accuracy of AUC (The Area Under the ROC curve) 0.85. (B) Colonic lavage predicted UC in accuracy of AUC 0.81. (C) The accuracy of AUC 0.88 increases when using data from stool and lavage

A

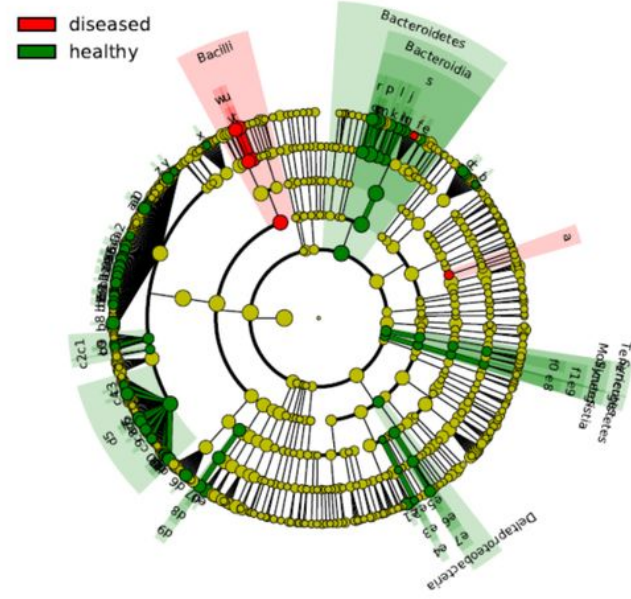

B

$\square$ diseased
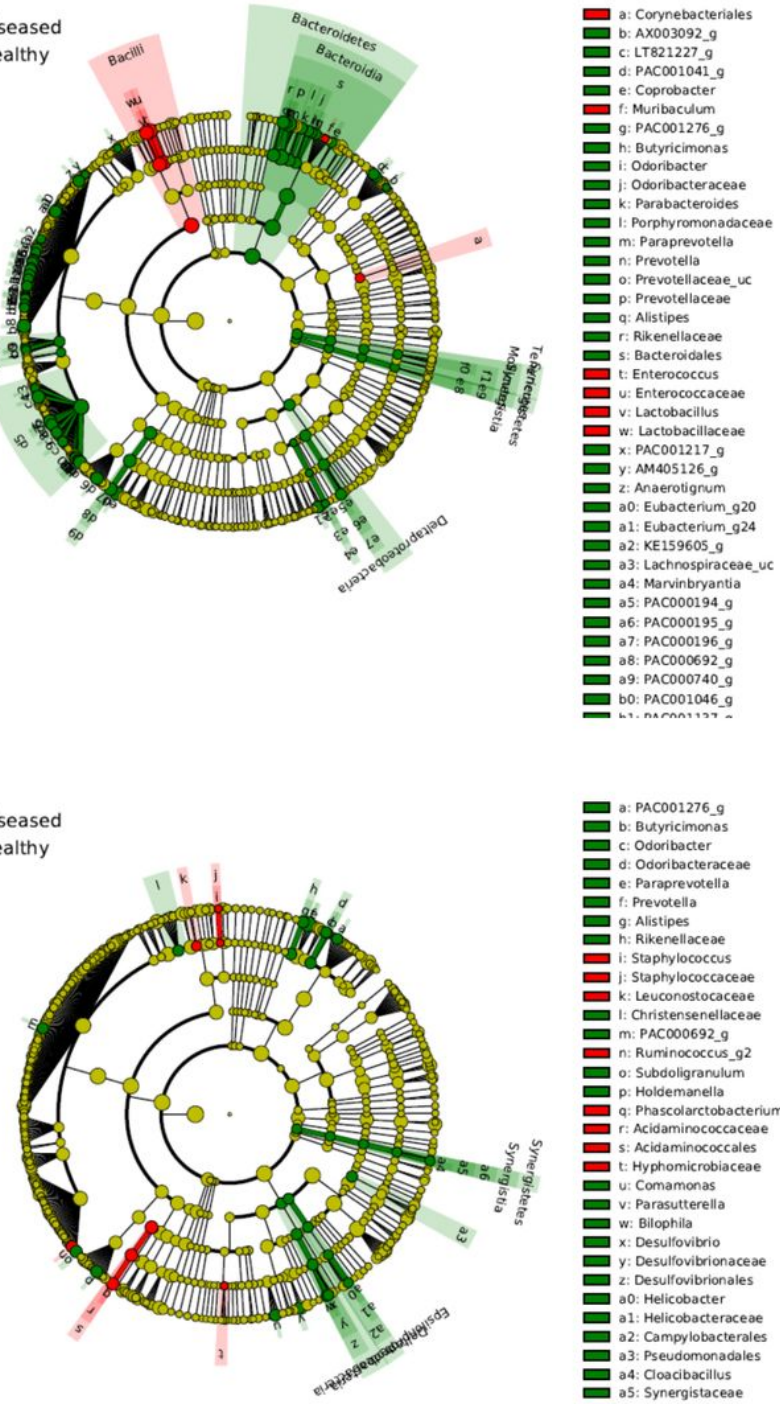

a: PAC001276_g

b: Butyricimonas

d: Odoribacter d: Odoribacterace f: Prevotella g: Alistipes h: Rikenellaceae i: Staphylococcus i: Staphylococcaceae i: Christensenellacea m: PACO00692.9. n: Ruminococcus_or o: Subdoligranulum p. Holdemanella q: Phascolarctobacterium s: Acidaminococcales t: Hyphomicrobiacea u: Comamonas vi Parasutterella w: Bilophila x: Desulfovibrio y: Desulfovibrionace a0: Helicobacter a1: Helicobacter a2: Campylobacterales a3: Pseudomonadales a4: Cloacibacillus as: Synergistaceae 


\section{Figure 6}

Cladogram generated by LEfSe indicating differences in taxa between ulcerative colitis group and control group. (A) In the case of stool, facultative anaerobes such as Enterococcus and Lactobacillus are UC-related biomarkers, and several species of Bacteroidetes (Prevotella, Bacteoides, etc.) and Clostridiales are found as markers of healthy control. (B) In case of lavage, Staphylococcus, Leuconostocaceae are the disease markers, and Prevotella, Alistipes, Desulfovibrio are the healthy control markers. 